\title{
Comercio de vino y lucha contra el fraude: Alicante entre el Antiguo
} Régimen y el Estado liberal ${ }^{1}$

\section{Wine Commerce and Fight against Fraud. Alicante during the Period Between the Ancient Regime and the Liberal Regime}

\author{
EDUARDO BUENO VERGARA \\ Universidad Miguel Hernández de Elche \\ ebueno@umh.es \\ ENRIQUE PERDIGUERO GIL \\ Universidad Miguel Hernández de Elche \\ quique@umh.es
}

\begin{abstract}
Resumen: Tomando como objeto de estudio la ciudad de Alicante, realizamos una aproximación a la lucha contra la adulteración del vino desde el final del Antiguo Régimen hasta la consolidación del régimen liberal. Durante la Edad moderna y las primeras décadas del siglo XIX, los esfuerzos estuvieron encaminados a evitar la entrada de vino "adulterado" con mezclas de otros vinos procedentes de fuera del término alicantino, en consonancia con las políticas proteccionistas. Una vez se decretó la libertad de comercio, en un contexto de crecimiento productivo y exportador, la adulteración fue objeto de un doble interés. Por un lado, se trató de perseguir la presencia de productos adulterantes en los vinos exportados, a fin de evitar el descrédito que eso suponía en el mercado internacional. Por otro, dentro de las preocupaciones higienistas, se trató de evitar que el uso de determinadas sustancias pudiera dañar la salud de la población.
\end{abstract}

Palabras clave: Agricultura, vino, comercio, seguridad alimentaria.

Abstract: Taking the city of Alicante as an object of study, we will make an approach to the fight against the adulteration of wine from the end of the Ancient Regime until the consolidation of the liberal regime. During the Modern Era and first decades of the nineteenth century, the efforts, along with protectionist policies, were aimed to prevent the entrance of the wine "adulterated" with blends of other wines coming from outside of

\footnotetext{
${ }^{1}$ Parte del contenido del presente artículo fue presentado por los autores en la comunicación "Control sanitario y comercio de vino en Alicante, España (siglo XIX)" en el marco del Congreso internacional Old and New Worlds: the Global Challenges of Rural History, celebrado en Lisboa entre el $27 \mathrm{y}$ el 30 de enero de 2016. El borrador de trabajo de dicha comunicación se puede consultar en: «https://lisbon2016rh.files. wordpress.com/2015/12/onw-0117.pdf» [consultado el 15 de mayo de 2017].
}

Recibido: 15 de mayo de 2017; aceptado: 9 de noviembre de 2017; publicado: 27 de marzo de 2018.

Revista Historia Autónoma, 12 (2018), pp. 133-150.

e-ISSN: 2254-8726; DOI: https://doi.org/10.15366/rha2018.12.007. 
the boundaries of Alicante. Once that free trade was decreed, in a production and trade growth background, adulteration was an object of double interest. One of them was to track the presence of adulteration products in exported wines in order to avoid the discredit that it entailed in the international market. Another matter of concern for the local hygienists was related to possible harmful effects of diverse substances in Alicante's population.

Keywords: Agriculture, Wine, Commerce, Food security.

\section{Objetivos}

El propósito del presente trabajo es acercarnos a la problemática que la adulteración del vino generó en un marco geográfico determinado, la ciudad de Alicante, y en un periodo cronológico que abarca la Edad Moderna - prestando especial atención al Setecientos - y también el siglo xIX, centrándonos en el control ejercido sobre su elaboración, comercio y consumo. Se trata de estudiar la evolución de la percepción de esa adulteración desde la etapa final del Antiguo Régimen hasta la llegada y consolidación del liberalismo, para analizar cómo cambiaron las necesidades de control y se articularon las nuevas respuestas dadas por la ciudad, teniendo en cuenta la relación existente entre la economía y el control alimentario, subrayando además el creciente valor dado a la protección de la salud de la población.

La pertinencia del estudio de un caso a partir del cual trascender la historia local viene dada por el valor estratégico que tuvo el vino para la economía alicantina ya desde la Edad Moderna y su participación en los circuitos comerciales internacionales. Al mismo tiempo, el ámbito local se convierte en un escenario privilegiado para analizar la dialéctica establecida con la administración central, teniendo en cuenta que el Estado fue desarrollando, progresivamente, una política económica nacional, al tiempo que participaba cada vez más en ámbitos relacionados con la salud de la colectividad, si bien es cierto que los municipios mantuvieron la iniciativa en las cuestiones sanitarias hasta bien entrado el siglo xx.

Las fuentes consultadas para la realización del trabajo proceden del Archivo Municipal de Alicante y han resultado especialmente útiles para el análisis del siglo XVIII y la primera 
mitad del XIX. Por otro lado, para las últimas décadas decimonónicas, la prensa local de la capital alicantina nos ha aportado un gran caudal de información a través de los diarios $E l$ Constitucional, El Liberal (ambos adscritos al Partido Liberal), El Graduador (órgano de expresión del Republicano Posibilista), La Unión Democrática (representante del Partido Republicano Progresista), La Provincia y El Eco de la Provincia (ambos defensores del Partido Conservador $)^{2}$. A través de estas publicaciones hemos tenido la oportunidad de comprobar, sobre todo, las preocupaciones de la burguesía alicantina, cuyos intereses económicos estaban centrados en la producción y la exportación vinícola.

\section{Introducción}

La vigilancia y las regulaciones efectuadas sobre los alimentos puestos a la venta pública en los centros urbanos surgieron desde el mismo momento que lo hicieron las ciudades. Su estudio ha formado parte de la llamada historia de la alimentación, una línea historiográfica que cuenta con una importante tradición desde los primeros estudios con metodología científica llevados a cabo en las décadas de 1920 y 1930 y que, después de la II Guerra Mundial fueron retomados con fuerza como parte del proyecto de historia total propuesta por la Escuela de Annales. En la actualidad, se trata de un objeto de estudio plenamente consolidado y abordado desde la pluralidad de enfoques que caracteriza la disciplina histórica ${ }^{3}$. Una de esas miradas corresponde al estudio de los cambios que se produjeron durante el siglo XIX en relación al control de la calidad de los alimentos frente al que se había mantenido en época preindustrial ${ }^{4}$.

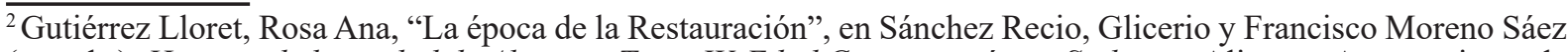
(coords.), Historia de la ciudad de Alicante. Tomo IV. Edad Contemporánea. Siglo XIX, Alicante, Ayuntamiento de Alicante, 1990, pp. 115-151; Moreno Sáez, Francisco, La prensa en la ciudad de Alicante durante la Restauración (1875-1898), Alicante, Instituto de Cultura Juan Gil-Albert, 1995.

${ }^{3}$ Grew, Raymond (ed.), Food in global history, Boulder, Westview Press, 2000; Kiple, Kenneth y Kriemhild Coneé Ornelas (eds.), The Cambridge World History of Food. Volume Two, Nueva York, Cambridge University Press, 2000; Super, John, "Food and History", en Journal of Social History, vol. 36, 1 (2002), pp. 165-178; Ferguson, Priscilla, "Eating Orders: Markets, Menus, and Meals", en The Journal of Modern History, vol. 77, 3 (2005), pp. 679-700; Spary, Emma, "Ways with Food”, en Journal of Contemporary History, vol. 40, 4 (2005), pp. 763-771; Scholliers, Peter, "Twenty-five Years of Studying un Phénomène Social Total", en Food, Culture \& Society, vol. 10, 3 (2007), pp. 449-471; Pérez Samper, María de los Ángeles, "La historia de la historia de la alimentación", en Chronica Nova, 35 (2009), pp. 105-162; Pilcher, Jeffrey (ed.), The Oxford Handbook of Food History, Nueva York, Oxford University Press, 2012; Uría González, Jorge, "Una nueva historia de la alimentación", en Historia contemporánea, 48 (2014), pp. 33-69.

${ }^{4}$ Filby, Frederick Arthur, A history of food adulteration and analysis, Londres, Allen \& Unwin, 1934; Teuteberg, Hans, "Food adulteration and the beginnings of uniform food legislation in late 19th century Germany", en Burnett, John y Derek Oddy (eds.), The Origins and development of food policies in Europe, Londres, Leicester University Press, 1994, pp. 146-160; Hardy, Anne, "Food, hygiene, and the laboratory. A short history of food poisoning in Britain, circa 1850-1950", en Social History of Medicine, vol. 12, 2 (1999), pp. 293-311; French, Michael y Jim Philips, Cheated not poisoned?: food regulation in the United Kingdom, 1875-1938, Manchester, Manchester University Press, 2000; Spiekermann, Uwe, "Food Quality in a Changing Social Environment: A Historical Perspective", en Grimme, Horst y Stefano Dumontet (eds.), Food Quality, Nutrition and Health. 5th Heidelberg Nutrition Forum/Proceedings of the ECBA, Berlín, Springer, 2000, pp. 37-48. DOI: https://doi. org/10.1007/978-3-642-59639-1_5; Law, Marc, "The Origins of State Pure Food Regulation”, en The Journal
} 
Estos cambios llegaron de la mano del proceso de industrialización aplicado a la producción, la distribución y el consumo de alimentos y del desarrollo de la química. Además, el crecimiento de la población y la rápida implantación de nuevos medios de transporte provocaron la apertura de redes de comercio internacionales, con el consiguiente aumento de la distancia entre productores y consumidores.

Las prácticas de sofisticación eran numerosas y se realizaban en casi cualquier tipo de producto. En general, el objetivo que se perseguía era obtener más cantidad de un alimento que era escaso o del que existía un abastecimiento insuficiente. Del mismo modo, se pretendía lograr que un producto fuese más atractivo a la vista, al olfato y al gusto a través de colorantes o potenciadores de sabor. Por último, se intentaba prolongar capacidad de conservación del producto o, incluso, practicar una suerte de "recuperación" cuando ya estaba echado a perder5.

Por lo que respecta al vino, en las décadas centrales del siglo XIX, la adulteración y el fraude se convirtieron en un problema fundamental para el sector. Estas prácticas se daban sobre la calidad — haciendo pasar vinos comunes por otros superiores - , sobre el origen — aprovechando una identificación geográfica - o mediante la adición de otros compuestos que alteraban las propiedades naturales e, incluso, los hacían nocivos para la salud ${ }^{6}$. A pesar de que, en un primer momento, gracias a este tipo de fraude era posible obtener beneficios rápidos y compensar los años de malas cosechas, la pérdida de confianza por parte de los consumidores provocaba una posterior reducción en el consumo o una caída del precio a medio plazo, algo que afectaba especialmente a los productores de vino de mayor calidad ${ }^{7}$.

Las cuestiones relacionadas con la adulteración del vino implicaron a un considerable número de agentes — productores, industrias asociadas, transportistas, comerciantes, consumidores, científicos, políticos, etc. — que demostraron intereses variados, pero también formó parte de la agenda exterior de los Estados dentro de sus políticas comerciales y diplomáticas. Estas preocupaciones cristalizaron en medidas legislativas y firmas de acuerdos comerciales internacionales por parte de los países, mientras que los productores trataron de certificar la

of Economic History, vol. 63, 4 (2003), pp. 1103-1130. DOI: https://doi.org/10.1017/S0022050703002547; Stanziani, Alessandro, "Negotiating Innovation in a Market Economy: Foodstuffs and Beverages Adulteration in Nineteenth-Century France", en Enterprise \& Society, vol. 8, 2 (2007), pp. 375-412; Atkins, Peter et al., Food and the City in Europe since 1800, Hampshire, Aldershot, 2007; Sanz Lafuente, Gloria, "Perspectivas de historia de la seguridad alimentaria. Entre la ley y la práctica social de la inspección 1855-1923”, en Revista española de estudios agrosociales y pesqueros, 212 (2006), pp. 81-118; Guillem Llobat, Ximo y Enrique Perdiguero Gil, "Control de la calidad de los alimentos y Urban Penalty: a propósito del caso valenciano (1881-1915)", en Historia Social, 80 (2014), pp. 113-131.

${ }^{5}$ Atkins, Peter, "Sophistication detected: Or, the adulteration of the milk supply, 1850-1914", en Social History, vol. 16, 3 (1991), pp. 317-339. DOI: https://doi.org/10.1080/03071029108567811.

${ }^{6}$ Stanziani, Alessandro, "La construction de la qualité du vin, 1880-1914", en Stanziani, Alessandro (dir.), La qualité des produits en France, XVIIIe-XXe siècle, París, Belin, 2004, pp. 123-150.

${ }^{7}$ Simpson, James, "Selling to reluctant drinkers: the British wine market, 1860-1914", en The Economic History Review, vol. 57, 1 (2004), pp. 80-108. DOI: https://doi.org/10.1111/j.0013-0017.2004.00273.x; Simpson, James, "Too Little Regulation? The British Market for Sherry, 1840-90", en Business History, vol. 47, 3 (2005), pp. 367-382. DOI: https://doi.org/10.1080/00076790500055988; Fernández, Eva, "Unsuccessful responses to quality uncertainty: Brands in Spain's sherry industry, 1920-1990”, en Business History, vol. 52, 1 (2010), pp. 100-119. DOI: https://doi.org/10.1080/00076790903469638. 
procedencia y calidad de sus vinos, lo que derivaría, ya en el siglo xx, en la vigilancia sobre los estándares de calidad a través de las marcas geográficas y las denominaciones de origen ${ }^{8}$. Por otro lado, se articularon medidas a nivel estatal, como el establecimiento de laboratorios aduaneros, mientras que, dentro de los organismos municipales, surgieron igualmente determinadas iniciativas a fin de intentar poner límites a la adulteración.

\section{Del Antiguo Régimen al liberalismo: cambio en la percepción de la adulteración}

El comercio de vinos a través del puerto constituía el pilar básico sobre el que se asentaba la economía alicantina desde la Edad Moderna, llegando a convertirse la vid prácticamente en monocultivo ${ }^{9}$ y posibilitando a las elites locales la acumulación de grandes fortunas vinculadas a la producción y exportación de caldos ${ }^{10}$. En términos absolutos, a finales del siglo XVIII, siempre dependiendo de la climatología que condicionaba la producción, se obtenían unos 300000 cántaros anuales (el cántaro equivalía a 11,55 litros), de los cuales podían embarcarse en torno a $90000^{11}$.

Durante la Edad Moderna existían fronteras exteriores, con otros estados, y también interiores, dentro de los propios territorios de la Monarquía, ambas con repercusiones arancelarias. En ese contexto y hasta las primeras décadas del siglo XIX, la entrada de vino en Alicante estuvo sujeta a una legislación de corte proteccionista: el Privilegio del vino, otorgado en 1510 por Fernando de Aragón y confirmado en diversas ocasiones con posterioridad. Su articulado establecía que únicamente estaba permitido vender y consumir vino elaborado en el término a partir de su uva, al tiempo que solo vecinos afincados en la ciudad podían adquirirlo

\footnotetext{
${ }^{8}$ Simpson, James, The Emergence of a Word Industry, 1840-1914, Princeton, Princeton University Press, 2011.

${ }^{9}$ Cernuda Juan, Enrique y Rafael Marhuenda Verdejo, Aspectos históricos de los vinos alicantinos, Alicante, Publicaciones del Instituto de Estudios Alicantinos, 1979; Giménez López, Enrique, Alicante en el siglo XVIII. Economía de una ciudad portuaria en el Antiguo Régimen, Valencia, Institución Alfonso el Magnánimo, 1981; Piqueras Haba, Juan, La vid y el vino en el País Valenciano. (Geografía económica), Valencia, Institución Alfonso el Magnánimo, 1981; Piqueras Haba, Juan, "La vid y el vino en Alacant: una síntesis histórica”, en Canelobre, 54 (2009), pp. 11-26; Alberola Romá, Armando, El pantano de Tibi y el sistema de riegos en la Huerta de Alicante, Alicante, Instituto de Cultura Juan Gil-Albert-Fundación CAM (2 $2^{a}$ ed. corregida y aumentada), 1994; Alberola Romá, Armando, "Propiedad, control y gestión del agua en regadíos deficitarios del sureste español: la Huerta de Alicante durante la Edad Moderna", en Minius. Historia, Arte e Xeografia, 23 (2015), pp. 7-40.

${ }^{10}$ Alberola Romá, Armando, Jurisdicción y propiedad de la tierra en Alicante (sS. XVII y XVIII), Alicante, Universidad de Alicante-Ayuntamiento de Alicante, 1984.

${ }^{11}$ La media del volumen de producción correspondiente a los últimos diez años del siglo es de 296319 cántaros. La producción total de vino durante el siglo XVIII ha quedado registrada en la serie documental de los Manifiestos del Vino, custodiada en Archivo Municipal de Alicante [en adelante, AMA], Armario 17, Libros 1-71. Estas fuentes han sido ya estudiadas en Giménez López, Enrique, Alicante en el... op. cit., pp. 428-429; Bueno Vergara, Eduardo, "Un indicador climático para el Alicante del siglo XVIII. Los manifiestos del vino", en Alberola Romá, Armando (coord.), Clima, naturaleza y desastre. España e Hispanoamérica durante la Edad Moderna, Valencia, Universitat de València, 2013, pp. 55-79.
} 
para su embarque ${ }^{12}$. El privilegio quedaría interrumpido en el momento en el que, dentro de la ciudad, la producción local estuviese prácticamente agotada y su precio de venta fuese considerado excesivo. Como es evidente, el objetivo de la medida era garantizar la ventaja de los productores locales frente a los forasteros, tanto en lo que se refiere al consumo interno, como a la exportación a través del puerto.

Con el fin de hacer cumplir el Privilegio se había creado desde mediados del siglo XVII una institución gobernada por cosecheros pertenecientes a la nobleza local, la llamada Junta de Inhibición del vino forastero. Esta organización estuvo dotada de la capacidad de emitir ordenanzas y bandos y mantuvo atribuciones ejecutivas que ejercía a través de un comisario que era elegido por sus miembros.

El control de la producción se realizaba mediante la llamada visita del vino. Una vez se había realizado la vendimia y se habían elaborado los vinos, una comisión compuesta por un síndico nombrado por el Ayuntamiento, un escribano y un maestro tonelero recorría todas las casas y bodegas tomando el manifiesto, es decir, recogiendo la cantidad de vino producido por cada propietario. Consignado el vino en posesión de cada cosechero, se contabilizaba el caldo que cada productor introducía en la ciudad, de modo que en ningún caso esta cantidad podía ser superior a la registrada durante la visita. Pese a ello, el fraude fue constante y el vino de otras localidades se introducía con frecuencia en Alicante, tal y como lo demuestran los numerosos pleitos judiciales que se incoaron por este motivo y que se conservan en el Archivo Municipal de la ciudad ${ }^{13}$. En total, para el siglo XVIII se conservan treinta y cuatro pleitos motivados por la introducción de vino forastero en la ciudad, encontrándose entre los procesados principalmente arrieros, pero también taberneros, bodegueros y comerciantes. En la mayoría de casos, el proceso se resolvía con la multa correspondiente al infractor, sin que se llevaran a cabo mayores averiguaciones $^{14}$.

La Junta se encargó de luchar a través de la vía administrativa por hacer prevalecer el privilegio y que este fuese confirmado por los monarcas, restringiendo en lo posible la entrada

\footnotetext{
${ }^{12}$ AMA, Armario 17, lib. 82 y lib. 86.

${ }^{13}$ Habida cuenta de la importancia del fraude durante la Edad Moderna, han sido abundantes y muy variados los enfoques que se han dado a su estudio, desde la normalización de estas prácticas, hasta la lucha contra las mismas, especialmente con la consolidación de las monarquías absolutas, pasando por la corrupción o el contrabando como medio de apropiación del patrimonio por parte de algunas elites, la fuente de ingresos que supuso la venalidad de cargos o la pluralidad jurisdiccional que favorecía la perpetuación del fraude. Ver Cárceles de Gea, Beatriz, "Fraude y administración fiscal en Castilla. La Comisión de Millones (1632-1658). Poder fiscal y privilegio jurídico-político", en Estudios de historia económica, 28 (1994), pp. 11-137; Cárceles de Gea, Beatriz, "Reforma y fraude fiscal en el reinado de Carlos II. La Sala de Millones (1658-1700)", en Estudios de historia económica, 31 (1995), pp. 9-154; Comín, Francisco, "El fraude fiscal en la Historia: un planteamiento de sus fases", en Hacienda pública española, 1 (1994), pp. 31-46; Rodríguez Gordillo, José Manuel, "El fraude en el estanco del tabaco (siglos XVII-XVIII)", en Hacienda pública española, 1 (1994), pp. 61-77, Yun Casalilla, Bartolomé, "Corrupción, fraude, eficacia hacendística y economía en la España del siglo XVII", en Hacienda pública española, 1 (1994), pp. 47-60; Jiménez Estrella, Antonio, "Poder, dinero y ventas de oficios y honores en la España del Antiguo Régimen: un estado de la cuestión", en Cuadernos de Historia Moderna, 37 (2012), pp. 259-271; Dubet, Anne, "Entre razón y ciencia de la Hacienda: la conflictiva construcción de un modelo de buen gobierno de la Real Hacienda en España en la primera mitad del siglo XVIII", en Espacio, tiempo y forma. Serie IV: Historia moderna, 28 (2015), pp. 187-209.

${ }_{14}^{14}$ AMA, Armario 3, caja 14, leg. 26; caja 22, leg. 13; caja 23, leg. 25.
} 
de producción forastera. Sin embargo, la aplicación de las medidas proteccionistas no estuvo libre de intentos por derogarla. Así, por ejemplo, en 1763, el entonces secretario de Hacienda, el Marqués de Esquilache, ordenó a la ciudad que no entorpeciese la libre entrada de vino para "uso y consumo de los militares enfermos existentes en ella" 15 , en 1772 se autorizó a los comerciantes desembarcar vino, siempre y cuando no se consumiese en Alicante y en 1796 se permitió su libre entrada durante los días de mercado. Por su parte, el Hospital Militar había logrado sortear el privilegio, contando con el permiso real para introducir vinos forasteros desde la década de 1720. En cualquier caso, a pesar de los intentos por acabar con el control comercial del vino que ostentaba la pequeña nobleza local, a principios del siglo XIX se mantuvo la legislación proteccionista, si bien es cierto que muy debilitada.

La importancia del factor proteccionista se compaginó con controles de tipo sanitario. Así, en agosto de 1781, a través del síndico personero se hacía saber al cabildo que se estaban despachando vinos adulterados "nocivos y perjudiciales para la salud pública"16. Una comisión formada por el fiel ejecutor, el síndico personero, los tres médicos contratados por la ciudad para la asistencia de los pobres ${ }^{17}$ y tres peritos — de los que no se especifica su profesión — se encargó de efectuar las averiguaciones pertinentes en todos los establecimientos en los que se despachaba vino. Tras un examen basado en las propiedades organolépticas, informó que se había encontrado una partida de vino adulterado cuyo consumo resultaba nocivo.

\section{El nuevo marco del liberalismo}

Desde las décadas finales del siglo XVIII y las primeras del siglo XIX, el sector vitivinícola español fue introduciendo rápidamente elementos y prácticas que podríamos calificar de industriales y que, necesariamente, cambiaron las necesidades de vigilancia del fraude que habían existido durante el Antiguo Régimen ${ }^{18}$. En el caso valenciano, una buena parte de su agricultura mantuvo una vocación hacia el comercio exterior durante el siglo XIX ${ }^{19}$, al tiempo que experimentaba una serie de transformaciones (cambio de cultivos, propiedad de la tierra), e introducía ciertas innovaciones tecnológicas que convivieron con otras prácticas tradicionales ${ }^{20}$.

\footnotetext{
$\overline{{ }^{15} \text { AMA, Armario }}$ 9, lib. 53, ff. 78v-79.

${ }^{16}$ AMA, Armario 9, lib. 76, ff. 260v-262.

${ }^{17}$ Perdiguero Gil, Enrique, "Con medios divinos y humanos: la lucha contra la enfermedad y la muerte en Alicante en el siglo XVIII", en Dynamis, 22 (2002), pp. 121-150.

${ }^{18}$ Pan-Montojo, Juan, "Las industrias vinícolas españolas: desarrollo y diversificación productiva entre el siglo XVIII y 1960", en Barciela López, Carlos y Antonio di Vittorio (eds.), Las industrias agroalimentarias en Italia y España durante los siglos XIX y XX, Alicante, Universidad de Alicante, 2003, pp. 313-334.

${ }^{19}$ Piqueras Haba, Juan, La agricultura valenciana de exportación y su formación histórica, Madrid, Instituto de Estudios Agrarios, Pesqueros y Alimentarios, 1985.

${ }^{20}$ Garrabou, Ramón, Un fals dilema. Modernitato endarreriment de l'agricultura valenciana (1850-1900), Valencia, Institució Alfons el Magnànim, 1985; Calatayud Giner, Salvador et al., "Les transformacions de la societat agrària
} 
En la ciudad de Alicante, las aportaciones de capital extranjero a través de los comerciantes afincados en la ciudad propiciaron la concentración importantes porciones de tierra dedicadas a la vid se fueron consolidando a medida que avanzaba el siglo XVIII, al tiempo que en el núcleo urbano adquirían viviendas y almacenes con fines comerciales ${ }^{21}$. De forma paralela, los grandes cosecheros se fueron convirtiendo en receptores de la producción de pequeños propietarios o arrendadores de tierras que no disponían de instalaciones (lagar, bodegas) para elaborar sus propios vinos ${ }^{22}$.

La progresiva implantación del régimen liberal creó nuevas tensiones entre las políticas proteccionistas y librecambistas que afectaron al control sobre el fraude del vino. De este modo, en noviembre de 1831 se acabó con el tradicional bando de la vendimia ${ }^{23}$, mientras que en febrero de 1834, mediante un Real Decreto, se estableció la libre circulación y compraventa de vinos en el territorio nacional satisfaciendo los derechos establecidos ${ }^{24}$.

En Alicante, los cambios acaecidos fueron bien recibidos por parte de grandes productores y comerciantes de vino, aunque demandaban mayor profundidad en las disposiciones de corte liberal, según recoge en 1837 la Memoria sobre vinos remitida al gobierno central ${ }^{25}$. La memoria recogía una serie de demandas de tipo librecambista para potenciar el sector del vino y se señalaba que las barreras al crecimiento se debían, sobre todo, a los "exorbitados derechos de consumo" municipales. Por ello, se proponía la eliminación del derecho de puertas, es decir, el pago de una cantidad por introducir vino en la ciudad "por ser de todo punto incompatible con un sistema liberal de gobierno y con la prosperidad nacional"26. A través de este informe se solicitaba la firma de tratados comerciales con otros Estados, a fin de facilitar la exportación vinícola al extranjero. Del mismo modo, se aludía a la necesidad de reducir los derechos existentes sobre las industrias aparejadas a la vinícola, como la maderera. En cuanto a otras consideraciones, se apuntaba la necesidad de mejorar las infraestructuras viarias a fin de hacer más transitables los caminos.

Todas estas demandas iban en la línea de las que durante los años centrales del siglo XIX mantuvo la burguesía alicantina. Las reivindicaciones quedaron perfectamente reflejadas en la publicación El Mensajero, el órgano de expresión de la Compañía alicantina de Fomento. Esta entidad, que reunía a hacendados y comerciantes, tenía como objetivo contribuir al desarrollo

en el procés de desenvolupament capitalista: el regadiu valencià el segle XIX", en Recerques: Història, economia $i$ cultura, 25 (1992), pp. 125-138; Mateu Tortosa, Enric y Salvador Calatayud Giner, "La Evolución de la agricultura valenciana: algunos aspectos (1840-1930)", en Vidal, Javier et al. (eds.), De la sociedad tradicional a la economía moderna: estudios de economía valenciana, Alicante, Instituto Alicantino de Cultura Juan Gil-Albert, 1996, pp. 101-120.

${ }^{21}$ Giménez López, Enrique, "La burguesía mercantil y la propiedad en el siglo XviII. El caso de Alicante”, en Alberola Romá, Armando y Emilio La Parra López (eds.), La Ilustración Española. Actas del Coloquio Internacional celebrado en Alicante, 1-4 octubre 1985, Alicante, Instituto Juan Gil-Albert, 1986, pp. 477-496; Piqueras Haba, Juan, La agricultura ... op. cit.

${ }^{22}$ Giménez López, Enrique, Alicante en el... op. cit.

${ }^{23}$ La Gaceta, 13 de diciembre de 1831.

${ }^{24}$ La Gaceta, 27 de febrero de 1834.

${ }^{25}$ AMA, Finanzas, leg. 2/55.

${ }^{26}$ Ibídem. 
económico dentro de las propuestas agraristas y librecambistas ${ }^{27}$. Entre los aspectos relativos al cultivo y exportación del vino a los que se refirió la publicación, encontramos la excesiva carga fiscal para propietarios y arrendatarios, los elevados impuestos municipales y derechos de puertas, o la falta de acuerdos comerciales internacionales que fomentasen la exportación ${ }^{28}$.

Teniendo en cuenta esta serie de transformaciones, el sentido de la adulteración del vino también hubo de adaptarse a esta nueva realidad productiva y comercial. De este modo, mientras estuvo vigente la legislación proteccionista, la adulteración - término habitualmente empleado en las fuentes - se refería sobre todo a la mezcla de vino alicantino con otro forastero, una práctica con la que se pretendía introducir caldos producidos fuera del término alicantino y que era perseguida por los oficiales de la Junta de inhibición del vino. Sin embargo, con el cese de las restricciones a la entrada de vinos foráneos en Alicante, la concepción de adulteración entendida exclusivamente como mezcla dejó de tener sentido. A partir de mediados del siglo xIX y, muy especialmente, en el último tercio de la centuria ${ }^{29}$, la adulteración se asoció exclusivamente a la adición de productos que alteraban las condiciones consideradas naturales del vino.

Durante la primera mitad del siglo no parece que la presencia de productos químicos en la cadena alimentaria se considerase un problema sanitario ${ }^{30}$. En un informe elaborado por la Comisión permanente de Salubridad municipal, fechado en 1849, se recogían los principales problemas que hacían peligrar la salud pública en la ciudad de Alicante ${ }^{31}$. En este detallado documento no se mencionaba la falsificación del vino ni de cualquier otro alimento con productos nocivos para la salud.

\section{La adulteración del vino en el contexto de auge comercial}

En el último tercio de la centuria, la importancia del comercio de vinos a través del puerto alicantino estaba plenamente consolidada y suponía una tercera parte del valor de todas las

\footnotetext{
${ }^{27}$ Cuevas, Joaquín, "La financiación del desarrollo agrario valenciano, 1750-1914”, en Historia Agraria, 25 (2001), pp. 89-120; Díaz Marín, Pedro, “Actividades y estrategias económicas de la burguesía alicantina en los años cuarenta del siglo XIX", en Investigaciones de Historia Económica, vol. 5, 15 (2009), pp. 137-170. DOI: https://doi.org/10.1016/S1698-6989(09)70122-5.

${ }^{28}$ Díaz Marín, Pedro, "Burgesia i ciutat. La política urbana a Alacant durant la Dècada Moderada (1844-1854)", en Recerques: Història, economia i cultura, 36 (1998), pp. 81-108; Díaz Marín, Pedro, Después de la Revolución. Centralismo y burguesía en Alicante, 1844-1854, Alicante, Generalitat Valenciana e Instituto de Cultura Juan GilAlbert, 1998.

${ }^{29}$ Guillem Llobat, Ximo, De la cuina a la fàbrica. L'aliment industrial i el frau. El cas valencià en el context internacional, Alicante, Universidad de Alicante, 2010.

${ }^{30}$ Salort i Vives, Salvador, "La lucha contra la enfermedad y la muerte. Inversiones públicas en salud en la ciudad de Alicante (1859-1923)”, en Boletín de la Asociación de Geógrafos Españoles, 48 (2008), pp. 327-354; Salort i Vives, Salvador, "Los inicios del municipio providencial en España como factor de modernización de las ciudades. Condiciones de vida, urbanismo, alimentación y salud en el modelo de la ciudad de Alacant/Alicante (18601923)", en del Arco Blanco, Miguel Ángel et al. (eds,), Ciudad y modernización en España y México, Granada, Universidad de Granada, 2013, pp. 103-126.

${ }^{31}$ AMA, Sanidad, leg. 2/3.
} 
exportaciones realizadas. En la década de 1880 ya representaba un 70\% de las mercancías, una situación que se mantuvo hasta principios del siglo $\mathrm{xx}^{32}$. Mientras que a finales del Setecientos se embarcaban en torno a 15000 hectólitros de vino al año ${ }^{33}$, en la última década del siglo XIX las exportaciones alcanzaban los 2500000 hectólitros, en cargamentos que salían desde el puerto de Alicante.

Con el tiempo se fue introduciendo en el sector vinícola el uso de industrias auxiliares que fueron ganando complejidad tecnológica hacia finales del siglo, dando entrada a diferentes elementos como máquinas prensadoras, aparatos para calentar o refrigerar los vinos, o bombas para comunicarlos de unos recipientes a otros. Además, actuó como motor de sectores asociados, como la tonelería o los aparejos de bodega. En un informe fechado en diciembre de 1883, enviado por el Ayuntamiento de Alicante al Gobernador Civil de la provincia, se hacía notar la importancia que había adquirido la tonelería, empujada por el auge comercial de vino ${ }^{34}$.

En este marco general, la adulteración de caldos, junto con la de muchos otros alimentos, despertaba una serie de preocupaciones, comerciales y sanitarias, que fueron recogidas por la prensa local. En lo que respecta al vino, El Constitucional denunciaba los perjuicios que provocaba en el consumidor la práctica de adulterarlo para darle fuerza y mejorar su conservación, o bien para modificar su color y "hacerle agradable a la vista, por más que altere la salud del que lo bebe" ${ }^{35}$. Teniendo en cuenta la importancia del vino en el contexto de la sociedad alicantina decimonónica, fueron dos los ejes sobre los que giró el control sanitario de este producto durante la segunda mitad del siglo. Por un lado, la vertiente comercial exterior requería del vino un prestigio capaz de borrar la sombra de la sospecha del fraude para hacerlo atractivo en el mercado. Por otro lado, dentro de una creciente preocupación general por la higiene y la salud pública, se trató de evitar el consumo de alimentos adulterados, entre ellos el vino, puesto que podía causar daño a la población ${ }^{36}$.

\subsection{El mantenimiento del prestigio internacional}

La burguesía alicantina tenía un interés evidente en asegurar el dinamismo del comercio vinícola, algo que quedó perfectamente reflejado en los periódicos, órganos de expresión de las diferentes fuerzas políticas, que se esforzaron en poner de manifiesto la necesidad de luchar contra las adulteraciones que podían dañar el prestigio de los caldos y, por consiguiente, dificultar sus exportaciones. En otras áreas geográficas peninsulares también existió este problema y, por

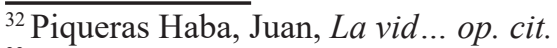

${ }^{33}$ Giménez López, Enrique, Alicante en el... op. cit.; Piqueras Haba, Juan, "La vid..." op. cit.

${ }^{34}$ AMA, Abastecimiento, leg. 92/16.

${ }^{35}$ El Constitucional, 5 de marzo de 1874, p. 2.

${ }^{36}$ Alessandro Stanziani, a través de los estudios que ha llevado a cabo, centrados en la Francia de los siglos XIX $\mathrm{y} \mathrm{xx}$, ha puesto de manifiesto que las cuestiones relacionadas con la adulteración de los vinos no responden únicamente a unos intereses estrictamente sanitarios, ver Stanziani, Alessandro, "Negotiating innovation..." op. cit.; Stanziani, Alessandro, "Information, quality and legal rules: Wine adulteration in nineteenth century France", en Business History, vol. 51, 2 (2009), pp. 268-291. DOI: https://doi.org/10.1080/00076790902726616.
} 
ejemplo, se ha identificado la adulteración de los vinos como una de las razones que redujo las exportaciones de Jerez a Gran Bretaña en las décadas finales del siglo XIX ${ }^{37}$.

Así, en agosto de 1874, El Constitucional señalaba la importancia de recobrar un prestigio perdido por culpa de las habituales adulteraciones que se atribuían a los vinos nacionales, aunque no podía ignorarse que alguna de estas acusaciones - consideraban - formaba parte de una estrategia interesada de los comerciantes ingleses y exportadores franceses para frenar la producción española ${ }^{38}$. En el mismo sentido se expresaba El Graduador, al lamentar el fuerte retroceso que se había experimentado en el comercio. Se exponía que el motivo por el que se había producido tal recesión no era otro que la pérdida de reputación de los productos embarcados en el puerto alicantino que "causa pavor y espanto en los demás mercados, por las repetidas veces que algunos comerciantes y productores han engañado al comercio de aquellos"39.

Aunque en la prensa alicantina el tema de los tratamientos químicos apenas había empezado a trascender, se afirmaba que en Francia era una práctica muy extendida que ya había causado gran alarma social ${ }^{40}$. La mezcla de vinos con otras sustancias era muy frecuente, como el conocido "enyesado", que consistía en la adición de yeso para contrarrestar defectos en la fermentación. Fue este uno de los procesos de adulteración que se dio con mayor frecuencia en el país galo y cuya regulación a finales del siglo XIX fue resultado de la negociación entre partidarios del uso del yeso, principalmente vinateros del Mediodía francés, y quienes se oponían al mismo, higienistas y productores de Burdeos ${ }^{41}$.

También se podía dar mayor estabilidad a través de diferentes conservantes o mediante el encabezado con otros vinos de mayor graduación o alcoholes no vínicos. En otros casos, se empleaban tintes naturales o artificiales para mejorar el color de los caldos ${ }^{42}$. Entre estos últimos, en el caso alicantino se mencionan el arrayán, el tornasol, el palo de Campeche, el palo de Pernambuco, la cochinilla, los pétalos de amapola, las cerezas, la flor de malva real o las bayas de mirto ${ }^{43}$. Sin embargo, el elemento más empleado para la mezcla fue la fucsina, un colorante de bajo coste que, en pequeñas dosis, dotaba a los caldos de "un tinte hermoso muy parecido al que generalmente tienen los vinos nuevos superiores"44, cuyo uso se había generalizado desde la década de $1860^{45}$. En la preparación de la fucsina, muchas veces quedaban trazas de arsénico, sustancia venenosa, pero aún en los casos en los que no contaba con este elemento, se discutía si era igualmente tóxica. Así, según un informe remitido a la Academia

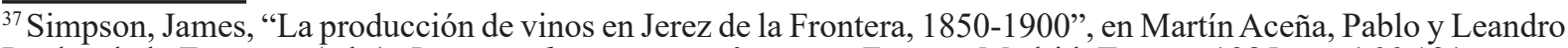
Prados de la Escosura (eds.), La nueva historia económica en España, Madrid, Tecnos, 1985, pp. 166-191.

${ }^{38}$ El Constitucional, 7 de agosto de 1874, p. 1.

${ }^{39}$ El Graduador, 29 de noviembre de 1876, p. 1.

${ }^{40}$ El Constitucional, 26 de julio de 1876, p. 2.

${ }^{41}$ Stanziani, Alessandro, "Negotiating innovation..." op. cit.

${ }^{42}$ Pan-Montojo, Juan, "El vino y la política comercial en la Restauración”, en Agricultura y Sociedad, 72 (1994), pp. 167-204.

${ }^{43}$ El Liberal, 19 de marzo de 1887, p. 2; Manero Mollá, Evaristo, Estudios sobre la Topografía Médica de Alicante, Alicante, Imprenta de Carratalá y Gadea, 1883.

${ }^{44}$ El Constitucional, 26 de julio de 1876, p. 2.

${ }^{45}$ El Constitucional, 5 septiembre de 1877, p. 2.
} 
de Medicina de Montpellier en abril de 1876 y del que se hacía eco la prensa alicantina, la ingesta de la fucsina, aun sin su componente arsenical, provocaba "albuminerio [...] una de esas enfermedades más terribles para la humanidad" 46 . Además, a través de experimentos en animales y humanos llevados a cabo en la Universidad de Nancy y divulgados en 1877, se había certificado la toxicidad de la fucsina, aunque fuese en pequeñas dosis, puesto que la sustancia se acumulaba en el cuerpo ${ }^{47}$.

Los periódicos locales se mostraban enormemente interesados en las noticias relacionadas con la coloración artificial de los vinos llegadas desde países del entorno. En julio de 1876, El Constitucional ya se hacía eco del empleo de fucsina en el mercado interior francés y cómo habían surgido demandas para actuar con severidad contra el fraude ${ }^{48}$. Los sucesos referidos a la detección de fucsina en partidas de vino recibieron mucha atención, así como los avances logrados en la lucha contra la adulteración, que servían para señalar los pasos que debían seguirse en el Estado español. Así, por ejemplo, a finales de 1876, se informaba de la decisión tomada en Italia de examinar en las aduanas los vinos importados y, en caso de resultar sospechosos, proceder a su análisis en busca de adulterantes ${ }^{49}$.

Con el paso de los años, los controles químicos en los laboratorios de las aduanas se fueron generalizando en Europa. En España, en 1879 se estableció un procedimiento estandarizado para la detección de la fucsina ${ }^{50}$, que fue sustituido un año más tarde como consecuencia de la incertidumbre que arrojaban sus resultados ${ }^{51}$. En Alicante, este tipo de indagaciones para detectar partidas de vino adulterado en los laboratorios de aduanas se venían realizando, al menos, desde $1881^{52}$.

En el marco de esa acción estatal contra la fucsina, una circular enviada por el Ministerio de Gobernación en febrero de 1879 recogía precisamente esta preocupación por la reputación de los caldos españoles. Se abundaba en el descrédito que estaba ocasionando el empleo de fucsina, "sustancia nociva y perjudicial a la salud", como colorante artificial en los vinos. Se instaba a los gobernadores a que, por todos los medios posibles, persiguieran y condujeran ante los tribunales a los adulteradores de vinos, tanto aquellos destinados a la exportación como al consumo interior, aunque no contemplaba novedades relativas a los mecanismos para detectar sustancias químicas ${ }^{53}$.

Sin embargo, en la década de 1880 la preocupación por la adulteración con fucsina fue disminuyendo. Las llamadas a los productores para que mantuvieran la reputación de los vinos, las demandas al legislador para que persiguiera a los responsables de la adulteración y los

\footnotetext{
$\overline{{ }^{46} \text { El Graduador, }} 16$ de diciembre de 1876, p. 1. La intoxicación por fucsina puede provocar la ruptura de los glóbulos rojos y, como consecuencia, presencia de albúmina en sangre.

${ }_{47}$ Pan-Montojo, Juan, La bodega del mundo: la vid y el vino en España, 1800-1936, Madrid, Alianza, 1994.

${ }^{48}$ El Constitucional, 27 julio de 1876, p. 2.

${ }^{49}$ El Constitucional, 2 de diciembre de 1876, p. 2.

${ }^{50}$ La Gaceta, 28 de marzo de 1879.

${ }^{51}$ La Gaceta, 9 de mayo de 1880.

${ }^{52}$ La Unión Democrática, 11 de enero de 1881, p. 2.

${ }^{53}$ La Gaceta, 28 de febrero de 1879, p. 581.
} 
artículos que informaban de las prácticas fraudulentas más habituales, desaparecieron de las páginas de las cabeceras alicantinas. En este periodo, sería la adulteración de los productos de consumo interno la que centraría mayoritariamente el interés de la burguesía alicantina. Este hecho hay que relacionarlo con una coyuntura comercial favorable, al menos hasta el cambio de tendencia experimentado a finales de la década de 1880. Por otro lado, la generalización de los análisis en las aduanas y el perfeccionamiento de las técnicas empleadas para la detección de la fucsina, a pesar de las incertidumbres, lograron limitar el uso de este colorante artificial.

Respecto al comercio exterior, Francia era el principal mercado para los vinos españoles que, por su elevada graduación alcohólica, eran empleados mayoritariamente para encabezar los franceses. Debido al descenso en la producción de vinos franceses a mediados de los setenta provocado por la filoxera ${ }^{54}$, el país galo se vio obligado a aumentar el volumen de importación para mantener la actividad del sector. La firma de acuerdos comerciales entre Francia y España ${ }^{55}$ rebajaron los aranceles e hizo más ventajosa la exportación de vinos a territorio francés ${ }^{56}$. De este modo, se creó una coyuntura económica extraordinaria en la que el incremento del volumen de las exportaciones y el aumento de los precios benefició enormemente al sector vitivinícola nacional, una situación que se prolongó hasta finales de la década de 1880 y principios de la siguiente ${ }^{57}$.

En Alicante, esta situación de bonanza tuvo su reflejo en un evidente optimismo por parte de la burguesía local que dio inicio a una serie de proyectos de embellecimiento de la ciudad, apertura de vías y ampliación urbanística, como en el caso del barrio de Benalúa, planeado a partir de los principios del ensanche higienista decimonónico ${ }^{58}$. En los años siguientes continuaron planes vinculados al abastecimiento de agua, la red de tranvías, la electricidad o la construcción ${ }^{59}$. En cualquier caso, toda esta actividad no solo se explica a partir de la bonanza económica derivada de la exportación del vino, sino que también influyeron otros factores, tales como una cierta estabilidad institucional, la ausencia de guerras, o la propia apuesta personal de las oligarquías urbanas por invertir y especular con proyectos de construcción y de prestación de servicios.

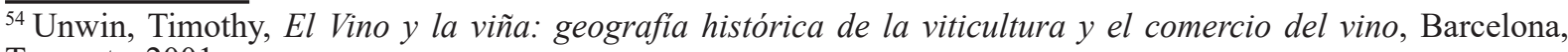
Tusquets, 2001.

${ }^{55}$ La Gaceta, 13 de mayo de 1882.

${ }^{56}$ Pan-Montojo, Juan, "El vino y..." op. cit.

${ }^{57}$ Pan-Montojo, Juan, La bodega ... op. cit.; Colomé Ferrer, Josep, “El sector vitícola español durante la segunda mitad del siglo XIX y el primer tercio del Xx: el impacto de la demanda francesa, la crisis ecológica y el cambio técnico", en Carmona Pidal, Juan et al. (eds.), Viñas, bodegas y mercados. El cambio técnico de la vitivinicultura española, 1850-1936, Zaragoza, Universidad de Zaragoza, 2001, pp. 43-58.

${ }^{58}$ Pérez del Hoyo, Raquel, "Benalúa de Alicante: la identidad de un barrio transformado reconsiderada desde la actualidad", en Arquitectura y Urbanismo, vol. 33, 2 (2012), pp. 24-46; Pérez del Hoyo, Raquel y María Elia Gutiérrez Mozo, "El sueño de un barrio y la problemática del ensanche: la obra inconclusa de Benalúa de Alicante (1883-1896)", en Pérez del Hoyo, Raquel (coord.), Apuntes en torno a la Arquitectura, Alicante, Universidad de Alicante, 2012, pp. 9-58.

${ }^{59}$ Vidal Olivares, Javier, "Burguesía y negocios: la especulación en el sector servicios de la ciudad de Alicante a fines del siglo XIX (1880-1900)", en Anales de la Universidad de Alicante. Historia Contemporánea, 2 (1983), pp. $159-181$
} 


\subsection{El vino como parte de la salud pública de la ciudad}

El otro eje sobre el que giró la preocupación por la adulteración vinícola fue la incidencia que el consumo de productos nocivos pudiera tener sobre a la población, un problema sanitario muy extendido y compartido por buena parte de las ciudades españolas ${ }^{60}$. Del mismo modo que sucedió con el caso de las exportaciones, este fue un problema que no se manifestó con intensidad hasta la séptima década de la centuria. En 1870 un detallado informe del concejal del arrabal de San Antón, construido extramuros y habitado mayoritariamente por las clases populares, señalaba que los vecinos de ese barrio no se abastecían en la plaza del mercado, sino en tiendas cercanas, en las que los precios eran más elevados y los géneros se solían encontrar adulterados por los tenderos ${ }^{61}$.

También a través de la prensa local hemos podido comprobar que el fraude alimentario era una práctica muy generalizada ${ }^{62}$, aunque el consumo cotidiano de vino adulterado no recibió tanta atención en sus páginas como los asuntos relativos al comercio internacional. Aunque entre las páginas de la prensa local sí aparecen mencionados los peligros que entrañaba el consumo de vino manipulado, pocas veces se trataba con detenimiento y la mayor parte de las veces era abordado dentro del contexto de las normas de higiene establecidas para el resto de alimentos ${ }^{63}$.

Durante el siglo XIX, el estado central fue haciéndose cargo de las preocupaciones derivadas del fraude alimentario ${ }^{64}$, si bien es cierto que estas cuestiones eran acometidas principalmente a través de la administración municipal. En el caso alicantino, los asuntos concernientes al abastecimiento recaían en la llamada "comisión de mercados". También disponía de diversos oficiales que ejercían el control de los alimentos como eran el fiel ejecutor, el fiel del represo o almotacén, los veedores especializados en trigo, carne, pescados y vino, incluso, en determinadas ocasiones, también se pedía el parecer de los médicos ${ }^{65}$. En el caso del vino,

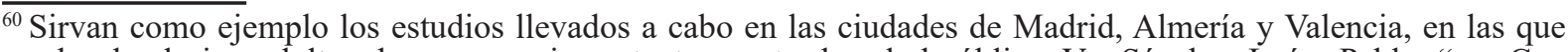
se aborda el vino adulterado como un importante asunto de salud pública. Ver Sánchez León, Pablo, "«... Con el nombre de vino»»": Alcoholes, fiscalidad y salud pública en el Madrid del siglo XIx", en Hacienda pública española, 158 (2001), pp. 191-214; Gómez Díaz, Donato y María José Gómez Díaz, "Control y fraude de los alimentos. Un viaje por la ciudad de Almería, 1788-1940”, en Martínez López, José Miguel (ed.), Historia de la alimentación rural y tradicional: recetario de Almería, Almería, Insituto de Estudios Almerienses, 2003, pp. 2951; Guillem Llobat, Ximo, De la cuina... op. cit.

${ }^{61}$ AMA, Armario 9, Actas del cabildo, sesión de 12 de febrero de 1870.

${ }^{62}$ El Graduador, 5 de julio de 1876, p. 2.

${ }^{63}$ Perdiguero Gil, Enrique y Josep Bernabeu Mestre, "Salud, alimentación y consumo", en Canelobre, 43 (20002001), pp. 104-117; Pascual Artiaga, Mercedes y Eva María Trescastro López, El desarrollo del municipio liberal y el reto de la alimentación en el Alicante de la primera mitad del siglo XIX, Alicante, Universidad de Alicante, 2013.

${ }^{64}$ Doval Pais, Antonio, Delitos de fraude alimentario: análisis de sus elementos esenciales, Pamplona, Aranzadi, 1996; Sanz Lafuente, Gloria, "Perspectivas de..." op. cit.; Pascual Artiaga, Mercedes y Eva María Trescastro López, El desarrollo... op. cit.

${ }^{65}$ Pascual Artiaga, Mercedes y Eva María Trescastro López, El desarrollo... op. cit.; Pascual Artiaga, Mercedes, "El reto de la alimentación en una ciudad mediterránea: Alicante en el siglo XIX", en Guillem Llobat, Ximo y Gabriel García Frasquet (eds.), Salud, Alimentació i cultura popular al País Valencià, Gandía, CEIC Alfons el Vell, 2009, pp. 151-168; Guillem Llobat, Ximo y Enrique Perdiguero Gil, “Control de la...” op. cit.
} 
a veces, se solicitaba la asistencia de maestros toneleros para comprobar que los recipientes de almacenamiento guardaban las medidas preceptivas. A medida que avanzó la centuria, la legislación local fue recogiendo las prevenciones referidas al control alimentario, dentro de las medidas denominadas de "policía de abastos", al tiempo que los bandos de la alcaldía ponían énfasis en la necesidad de velar por el cumplimiento de las normas.

No obstante, se hacía patente que no era posible controlar el fraude alimentario únicamente a través de los oficiales con los que contaba el Ayuntamiento. Su pericia se basaba en la percepción de las propiedades organolépticas, pero los retos introducidos por el empleo de sustancias químicas, muchas veces imperceptibles a los sentidos, hacían que los medios tradicionales no resultasen eficaces. Sin embargo, el médico higienista Evaristo Manero señalaba en sus Estudios sobre la Topografía médica de Alicante, que la mezcla de vinos con agua o la adición de alcohol de manera artificial no podía ser descubierta mediante procedimientos científicos, sino que "sólo el paladar de las personas inteligentes" podían descubrir el fraude ${ }^{66}$.

La principal medida llevada a cabo para combatir con el fraude alimentario fue la creación del Laboratorio Químico Municipal en $1887^{67}$, del mismo modo en que se había llevado a cabo en otras ciudades españolas. Así, Madrid contaba con un laboratorio químico desde $1878^{68}$, Valencia había instalado el suyo en $1881^{69}$ y Zaragoza lo hizo en $1886^{70}$. El establecimiento del gabinete químico fue saludado por todas las cabeceras locales y en él se debían realizar los análisis de una gran variedad de alimentos, prestándose especial interés al vino, con el objetivo de evitar la adulteración "con drogas para disimular averías o mala calidad”, como recogió el diario afín al gobierno de ese momento ${ }^{71}$.

Para el cargo de director del Laboratorio, fue elegido José Soler Sánchez, Catedrático de Física y Química en el Instituto de Segunda Enseñanza de la ciudad, miembro del partido Liberal y una de las figuras de mayor protagonismo en el seno de la burguesía alicantina de finales de $\operatorname{siglo}^{72}$. El prestigio científico de Soler, que había sido Catedrático de Química Inorgánica en la Universidad Central de Madrid antes de regresar a Alicante para hacerse cargo de la farmacia familiar, estaba fuera de toda duda, pero su participación en la política municipal — fue concejal

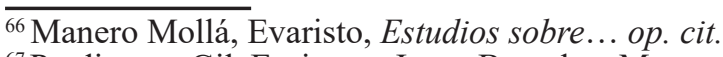

${ }^{67}$ Perdiguero Gil, Enrique y Josep Bernabeu Mestre, "Un reto a la modernización: el control de la enfermedad y de la muerte”, en Moreno Sáez, Francisco et al. (coords.), Los inicios de la modernización en Alicante, Alicante, Caja de Ahorros del Mediterráneo, 1999, pp. 137-156.

${ }^{68}$ Puerto Sarmiento, Francisco Javier y Josefa Cobo Cobo, "El Laboratorio Municipal de Madrid en el último tercio del siglo XIX”, en Dynamis, 3 (1983), pp. 149-172.

${ }^{69}$ Martínez, Felip et al. "La institucionalitzaciò de la salut pública a València: dels laboratoris químic i bacterológic a l'Institutit Municipal d'Higiene", en Barona Vilar, Josep Lluis y Juan Antonio Micó Navarro (coords.), Salut $i$ malaltia en els municipis valencians, València, Universitat de València, 1996, pp. 191-214.

${ }^{70}$ Sanz Lafuente, Gloria, "Perspectivas de..." op. cit.

${ }^{71}$ El Liberal, 2 de abril de 1887, p. 1.

${ }^{72}$ Rico García, Manuel, Ensayo Biográfico Bibliográfico de escritores de Alicante y su provincia, Alicante, Establecimiento tipográfico de Antonio Reus, 1889; Garcia Molina, Rafael, "José Soler Sánchez i altres hòmens de ciència alacantins", en Quaderns de Migjorn, 4 (2003), pp. 109-131.
} 
y alcalde interino durante 1882 - y su posterior nombramiento al frente del Laboratorio, lo convirtieron en centro de un enconado debate entre los principales diarios locales ${ }^{73}$.

En un primer momento, lejos de cuestiones sanitarias, el debate público giró en torno al posible uso partidista del gabinete. En este contexto, El Graduador apuntaba que el Laboratorio Químico podía ser utilizado como arma política, insinuando que los vinos levantarían sospechas solo dependiendo de la adscripción política del productor ${ }^{74}$. Por su parte, desde El Constitucional se hacían eco de las acusaciones de la prensa republicana y señalaban la gravedad de que el Laboratorio en lugar de ser "químico", fuese "político"75.

Las críticas arreciaron cuando los diarios de la oposición mostraron su incredulidad ante el hecho de que, en las primeras semanas de funcionamiento, no se hubiesen encontrado muestras de fucsina en los vinos, teniendo en cuenta lo extendida que estaba la práctica de colorear los vinos con esta sustancia, acusando al Ayuntamiento por su incapacidad para resolver esta importante cuestión para la salud pública ${ }^{76}$. En esta línea, se culpó a los responsables de la gestión del Laboratorio del secretismo con el que se trataban los resultados de los análisis y la desidia con la que actuaban ${ }^{77}$, unas acusaciones que siempre fueron replicadas desde el diario oficialista El Liberal $^{78}$.

Como es evidente, a la hora de analizar estas fuentes, no podemos pasar por alto el uso político que tuvo la lucha contra la adulteración de los alimentos y, en especial, la referida a un producto de tanto valor para la sociedad alicantina como era el vino. De este modo, la preocupación higienista por la salud de la población tuvo uno de sus reflejos en el enfrentamiento político a través de la prensa. Así, mientras que el periódico afín al partido que estaba en la alcaldía solía hablar en términos elogiosos de la gestión que se llevaba a cabo, desde la oposición no desaprovechaban ocasión para lanzar sus acusaciones, empleando la adulteración del vino como parte de la retórica del debate partidista.

A estas dificultades iniciales hubo que sumar las deficiencias presupuestarias que limitaron la capacidad para llevar a cabo los análisis. Para solucionar este problema se acordó que los particulares también pudieran solicitar la inspección de alimentos, previo pago de la tarifa correspondiente. Junto a esta medida, las multas impuestas a los propietarios de productos adulterados también sufragaron los gastos del Laboratorio. No menos importancia tuvo el prestigio del que gozaba el director, lo que contribuyó a asegurar el funcionamiento del gabinete y, a finales de siglo, el Ayuntamiento alicantino aprobó una nueva ordenanza en previsión de un aumento de la actividad. No obstante, al abandonar Soler la dirección, la institución sufrió

\footnotetext{
${ }^{73}$ García Belmar, Antonio, "El Laboratorio de la verdad. Peritaje químico, opinión pública y control de alimentos en el Laboratorio Químico Municipal de Alicante, durante el último tercio del siglo XIX", en Ballester Añón, Rosa et al. (eds.), Salud y enfermedad en la sociedad alicantina contemporánea, Alicante, Instituto Alicantino de Cultura Juan Gil-Albert, 2012, pp. 82-85.

${ }^{74}$ El Graduador, 4 de septiembre de 1887, p. 2.

${ }^{75}$ El Constitucional, 6 de septiembre de 1887, p. 2.

${ }^{76}$ El Constitucional, 8 de septiembre de 1887, p. 2.

${ }^{77}$ El Constitucional, 22 de septiembre de 1887, p. 2; El Constitucional, 30 de julio 1887, p. 2.

${ }^{78}$ El Liberal, 31 de julio de 1887, p. 3.
} 
una serie de altibajos, como su cambio de emplazamiento y una reducción presupuestaria, que pusieron en cuestión su continuidad ${ }^{79}$.

Un balance de la actividad del Laboratorio fue realizado en 1894 por Esteban Sánchez Santana, destacado higienista y médico colaborador de Soler, y José Guardiola, arquitecto municipal de Alicante, en su obra Memoria Higiénica de Alicante. Señalaban que, en los ocho años que llevaba establecido el gabinete químico, se habían analizado 1.068 muestras de alimentos, de las cuales 258 correspondían a vino, encontrándose adulteradas 64 de ellas. Según estos autores, a pesar de que no parecía que el nivel de adulteración fuera muy elevado, el hecho de que las muestras fueran recogidas por la guardia municipal, previo aviso a los comerciantes, indicaba que el nivel total de falsificación debía ser mucho mayor. No obstante, según su testimonio, gracias a las detecciones y multas impuestas se había reducido considerablemente la adulteración de productos desde la puesta en marcha del Laboratorio ${ }^{80}$.

\section{Conclusiones}

La lucha contra la adulteración de los vinos en la ciudad de Alicante durante los siglos XVIII y XIX fue el resultado, por un lado, de cuestiones comerciales $\mathrm{y}$, por otro, de argumentos vinculados a la salud pública. De este modo, el concepto de fraude varió en la medida en que lo hicieron los contextos y los intereses de determinados agentes. Así, durante la Edad Moderna, el fraude o la adulteración consistía fundamentalmente en la mezcla del vino con caldos procedentes de otros municipios, dentro de una tradicional política de privilegios que beneficiaba a los productores alicantinos. Aunque en todo momento se vigiló la inocuidad de los alimentos puestos a la venta, el control al que se sometía el vino para vigilar su adecuación para el consumo, en ningún caso recibió durante el Setecientos la misma atención por parte de las autoridades que la labor llevada a cabo por la poderosa Junta de Inhibición.

Con la llegada del liberalismo y la eliminación del andamiaje legal del Antiguo Régimen, la protección de la cosecha local dejó de tener sentido. Los nuevos desafíos aparecieron sobre todo en el último tercio de la centuria. En el plano comercial, el interés principal consistió en prevenir el fraude en las exportaciones para mantener un prestigio que facilitara la salida de las mercancías, algo que no siempre se consiguió y preocupó sobremanera a los productores locales de vino, inquietud que expresaban continuamente a través de diferentes periódicos. Una

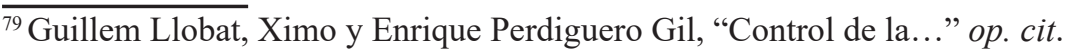

${ }^{80}$ Sánchez Santana, Esteban y José Guardiola Picó, Memoria Higiénica de Alicante. Escrita en virtud de Real Orden de 20 de Marzo de 1894, Alicante, Establecimiento Tipográfico de Costa y Mira, 1894.
} 
coyuntura económica muy favorable y la generalización de los análisis químicos llevados a cabo en las aduanas, hicieron que disminuyera dicha preocupación en la década de 1880 .

El segundo desafío estuvo ligado con el creciente valor otorgado a la salud pública, que fue ganando peso en la agenda política del Estado central, si bien es cierto que la administración municipal continuó desempeñando el papel principal en esta materia. Fue en ese contexto cuando, a finales del siglo, en sintonía con las propuestas higienistas, las necesidades de control del vino se materializaron en la creación del Laboratorio Químico Municipal, a cuyo frente se puso a un reputado científico. De esta manera, si bien la vertiente económica mantuvo su importancia, el elemento sanitario se convirtió en un factor fundamental a la hora de vigilar la adulteración del vino. 